\title{
Fully-Coupled Multi-Physical Simulation with Physics-Based Nonlinearity-Elimination Preconditioned Inexact Newton Method for Enhanced Oil Recovery
}

\author{
Huiying Tang ${ }^{1}$, Shihao Wang ${ }^{2, *}$, Congbin Yin ${ }^{3}$, Yuan $\mathrm{Di}^{4}$, \\ $\mathrm{Yu}-\mathrm{Shu} \mathrm{Wu}^{2}$ and Yonghong Wang ${ }^{5}$ \\ ${ }^{1}$ State Key Laboratory of Oil and Gas Reservoir Geology and Exploitation, Southwest \\ Petroleum University, Chengdu, P.R. China. \\ ${ }^{2}$ Petroleum Engineering Department, Colorado School of Mines, Golden, CO 80401, USA. \\ ${ }^{3}$ Chuanqing Downhold Service, Chengdu, P.R. China. \\ ${ }^{4}$ Department of Energy and Resources Engineering, Peking University, Beijing 100871, \\ P.R. China. \\ ${ }^{5}$ CNPC Engineering Technology RED Company Limited, Houston, Texas 77042, USA.
}

Received 12 May 2017; Accepted (in revised version) 16 December 2017

\begin{abstract}
In this paper, we introduce a physics-based nonlinear preconditioned Inexact Newton Method (INB) for the multiphysical simulation of fractured reservoirs. Instead of solving the partial differential equations (PDE) exactly, Inexact Newton method finds a direction for the iteration and solves the equations inexactly with fewer iterations. However, when the equations are not smooth enough, especially when local discontinuities exits, and when proper preconditioning operations are not adopted, the Inexact Newton method may be slow or even stagnant.

As pointed out by Keyes et al. [1], multi-physical numerical simulation faces several challenges, one of which is the local-scale nonlinearity and discontinuity. In this work, we have proposed and studied a nonlinear preconditioner to improve the performance of Inexact Newton Method. The nonlinear preconditioner is essentially a physics-based strategy to adaptively identify and eliminate the highly nonlinear zones.

The proposed algorithm has been implemented into our fully coupled, fully implicit THM reservoir simulator (Wang et al. $[2,3]$ ) to study the effects of cold water injection on fractured petroleum reservoirs. The results of this work show that after the implementation of this nonlinear preconditioner, the iterative solver has become significantly more robust and efficient.
\end{abstract}

AMS subject classifications: $60-08$

Key words: Physics-based nonlinearity-elimination, Inexact Newton method, thermal-hydraulicmechanical simulation, restricted additive Schwarz approach, parallel reservoir simulation.

\footnotetext{
*Corresponding author. Email addresses: gohych@gmail.com (S. Wang), appythy@gmail .com (H. Tang), cyin@chuanqing.com (C. Yin), diyuan@mech.pku.edu.cn (Y. Di), ywu@mines.edu (Y.-S. Wu), yonghong. wang@cnpcusa.com (Y. Wang)
} 


\section{Introduction}

Newton's method is a broad range of iterative methods for nonlinear programming. Starting from an initial guess, Newton's method computes the search direction based on the gradient of the given function. Once the search direction is determined, the searching step can be calculated by multiple approaches, including line-search and directly computing from residuals (Newton-Raphson method).

Exact Newton's method solves the problem iteratively until the residual is smaller than a pre-set criteria. In petroleum reservoir simulation, exact Newton-Raphson (NR) method is widely adopted. Inexact Newton's method (IN), on the other hand, solves the problem inexactly. Compared to exact Newton's method, IN requires less computation time, but may have poorer numerical stability. The Inexact Newton Method with Backtracking (INB) is an appealing approach for large scale numerical simulation. INB solves a nonlinear system approximately within each iteration. It can save much computational time spent that would otherwise be used for the linear solver. However, one of the drawbacks of the INB algorithm is that it is not as robust as Newton-Raphson algorithm (NR) in certain cases, as reported by other researchers [4-6]. For INB algorithm, it has been proven by Kelley [7] that if the target nonlinear equation is continuously differentiable and there is a limit point at which the Jacobian matrix is nonsingular, then the INB will converge at that limit point. With the existence of local discontinuities, the target equation is no longer continuously differentiable and the more the discontinuities, the farther the system is away from a continuously differential condition. Therefore as the number of local discontinuities increases, INB may suffer from convergence problems. Globalization techniques $[8,9]$ help improve the numerical performance of IN, but they cannot fully resolve the convergence issue, as reported by Knoll and Keyes [10].

For several types of reservoir simulation problems, such as water-oil displacement, nonlinearity exists only in a small portion of the entire field. This local nonlinearity will dramatically slow down the convergence of INB solvers. For the INB method, if the local nonlinearity is too high, the iteration will tend to be stagnant. Therefore, to guarantee the robustness of the INB, a nonlinear preconditioner is typically required, such as the Additive Schwarz Preconditioned Inexact Newton (ASPIN), as proposed by Cai and Keyes [11]. Additive Schwarz (AS) [12] method is essentially a domain decomposition approach. It subdivides the problem domain into several sub-domains and solves the sub-space problem separately in parallel. For non-overlapping AS, there is no communication among subdomains. For overlapping AS, neighboring subdomains exchange boundary conditions with each other. Cai and Sarkis [13] brought out the Restricted Additive Schwarz method, which has less communication among processors and better the numerical stability.

Recently, ASPIN has been applied to groundwater and oil simulation problems by (Skogestad et al. [14] and Liu et al. [15], respectively. ASPIN has been shown to be able to improve the convergence rate for highly heterogeneous reservoir simulation problems. As far as we know, there have very few attempts to adopt the nonlinearity elimination 
(NE) technique to precondition reservoir simulation problems that have high nonlinearity on parts of the computational domain. H. Yang et al. [16] used the active-set reducedspace method to eliminate the nonlinearity caused capillary pressure and relative permeability in two-phase flow which was different from this paper in both model and algorithmic point of view.

In this work, we propose another type of preconditioned INB method to deal with the local nonlinearity problems. We aim to eliminate the local discontinuity to enhance the numerical performance of the INB algorithm. Nonlinearity elimination techniques for INB have been studied by Hwang et al. $[17,18]$ with Computational Fluid Dynamics (CFD) problems. In this work, we will introduce an adaptive nonlinearity elimination (ANE) algorithm. This ANE algorithm adopts a physics based criterion to choose a highly nonlinear region out of the computational domain and eliminates the local nonlinearity before the start of INB. The highly nonlinear region can be adaptively chosen in each time step. Hwang et al. [18] first proposed such combined ANE-INB approach and applied it to the solution of the transonic full potential equation. In their work, the target equation is in steady state. In this work, we will implement ANE-INB into a transient reservoir simulation problem. We will investigate the performance of ANE-INB in the study of coupled THM behavior of fractured reservoirs, in particular for the elastic-plastic responses of fractures induced by cold water injection into oil/gas reservoirs. Moreover, as our simulator is massively parallel, we implement a restricted Schwarz nonlinear preconditioner to balance the nonlinearity. The restricted Schwarz preconditioner can be conveniently implemented in an existing code with little additional communication and computation.

\section{Problem description}

\subsection{Governing equations}

Firstly, to study the coupled thermal-hydraulic-mechanical (THM) processes of fractured reservoirs, a massively parallel simulator has been developed. The simulator is based on the framework of MSFLOW-MP [19] and is able to simultaneously solve the coupled thermal-hydraulic-mechanical behaviors of fractured reservoirs. The governing equation for hydraulic and thermal flow is as follows:

$$
\frac{d M^{k}}{d t}=\nabla \cdot \vec{F}^{k}+q^{k}
$$

In our simulator, $k=1$ refers to the hydrocarbon component; $k=2$ refers to the water component; $k=3$ refers to the heat (energy) component. In the above equation, $q$ is the sink/source term, $M$ is the conserved component in-place, and $F$ is the flux. For water/oil equations, the mass component in-place is

$$
M^{k}=\phi \sum_{\beta} S_{\beta} \rho_{\beta},
$$


where $\phi$ is the rock porosity; $S_{\beta}$ and $\rho_{\beta}$ are the saturation and density of phase, respectively. The flux term is given by

$$
\vec{F}_{\beta}^{k=1,2}=-K \frac{K_{r \beta} \rho_{\beta}}{\mu_{\beta}}\left(\nabla P_{\beta}-\rho_{\beta} g\right),
$$

where $K$ is the absolute permeability; $P_{\beta}$ is the pressure of phase $\beta ; K_{r \beta}$ and $\mu_{\beta}$ are the relative permeability and viscosity of phase $\beta$, respectively; and $g$ is the gravitational acceleration vector. The heat flux term includes conduction and convection,

$$
\vec{F}^{k=3}=-\left[(1-\phi) K_{R}+\phi \sum_{\beta} S_{\beta} K_{\beta}\right] \nabla T+\sum_{\beta} h_{\beta} \vec{F}_{\beta},
$$

where $K_{R}$ and $K_{b}$ are thermal conductivity of the rock and the liquid phase, $\beta$, respectively; $\vec{F}_{\beta}$ is the liquid flux term used in the oil/water equation; $T$ is temperature; and $h_{\beta}$ is enthalpy of liquid phase $\beta$. The accumulation term for the heat equation is the following,

$$
M^{\kappa}=(1-\phi) \rho_{R} C_{R} T+\phi \sum_{\beta} S_{\beta} \rho_{\beta} u_{\beta},
$$

where $\rho_{R}$ and $C_{R}$ are the density and heat capacity of rock, respectively, and $u_{\beta}$ is the internal energy of liquid phase $\beta$.

For the mechanical simulation, we start from the following displacement equation for a thermo-poroelastic material [3],

$$
\alpha \nabla P+3 \beta K \nabla T+(\lambda+G) \nabla(\nabla \cdot \vec{u})+G \nabla^{2} \vec{u}+\vec{F}=0,
$$

where $\alpha$ is the Biot's coefficient, $\lambda$ is the Lame's parameter, $G$ is the shear modulus, $\vec{F}$ is the body force and $\vec{u}$ is the displacement vector.

The normal stresses appearing in Hooke' law for a linear thermo-poro-elastic material are

$$
\sigma_{k k}-\left(\alpha P+3 \beta K\left(T-T_{r e f}\right)\right)=\lambda \varepsilon_{v}+2 G \varepsilon_{k k}, \quad k=x, y, z,
$$

where $\sigma_{k k}$ is the normal stress along the $k_{t h}$ direction, $\beta$ is the thermal expansion coefficient, $T_{r e f}$ is a reference temperature, and $\varepsilon_{v}$ is the volumetric strain, the summation of normal strain $\varepsilon_{k k}$ over the three directions

$$
\varepsilon_{v}=\varepsilon_{x x}+\varepsilon_{y y}+\varepsilon_{z z} .
$$

We introduce the mean stress $\sigma_{m}$ as follows,

$$
\sigma_{m}=\frac{\sigma_{x x}+\sigma_{y y}+\sigma_{z z}}{3}
$$

Then, after several steps of derivation [3], we arrive at the governing mean stress equation,

$$
\frac{3(1-v)}{(1+v)} \nabla^{2} \sigma_{m}+\nabla \cdot \vec{F}=\frac{2(1-2 v)}{(1+v)}\left(\alpha \nabla^{2} P+3 \beta K \nabla^{2} T\right) \text {. }
$$


The program solves two fluid flow equations, one thermal equation and one stress equation simultaneously within each time step. Therefore, the thermal-hydraulic-mechanical process is solved fully coupled. The primary variables are the water phase pressure, the water phase saturation, the temperature and the mean stress.

\subsection{Integral Finite Difference (IFD) method}

We use Integral Finite Difference (IFD) method to solve the governing equations. By integrating each PDE over a representative element volume (REV), we get the following integrated governing equation,

$$
\frac{d}{d t} \int_{V_{n}} M^{k} d V_{n}=\int_{\Gamma_{n}} \vec{F}^{k} \cdot \vec{n} d \Gamma_{n}+\int_{V_{n}} q^{k} d V_{n} .
$$

Using the IFD method, the governing equations are discretized as the following general form,

$$
\frac{\left(V^{i+1} M^{k, i+1}-V^{i} M^{k, i}\right)}{\Delta t}=\sum_{m} A_{n m} F_{n m}^{k}+q^{k} .
$$

The details of the simulator framework and this IFD implementation can be found in $[2,20]$. The above equation is solved by our proposed nonlinear solvers, to be described in a later session.

\subsection{Fracture aperture change induced by cold water injection}

When cold water is injected into fracture reservoirs, the matrix rock shrinks due to the thermal change and the fracture aperture, which is directly related to permeability, thus increases. Therefore, to quantify the above coupled thermal-hydraulic-mechanical effects is the key to the accurate prediction of the production performance of fracture reservoirs with cold water injection wells.

Ghassemi and Kumar [21] presented semi-analytical solution of the deformation of one single fracture in a geothermal reservoir subject to the similar THM process. Rutqvist et al. [22] and Kim et al. [23] developed sequentially/iteratively coupled numerical approaches to simulate the multiphysical effects. In this work, we use the semi-analytical correlation of the fracture aperture change proposed by Wang et al. [3], as follows. Consider the dual continua matrix-fracture system (Fig. 1). We focus on a matrix block surrounded by fractures of length $L_{i}$ on the $i$-th direction. The temperature and pressure in the fractures are kept constant as $P_{f}$ and $T_{f}$, respectively. The initial temperature and pressure of the matrix block are $P_{m 0}$ and $T_{m 0}$, respectively. For a 1-D problem, and ignoring leak-off into the matrix block, the temperature distribution inside the matrix rock can be solved analytically from the governing heat equation as [24]

$$
T_{m}=T_{f}+\sum_{n=1}^{\infty} \frac{4\left(T_{i n i}-T_{f}\right)}{(2 n-1) \pi} \sin \left[\frac{(2 n-1) \pi}{L_{i}} x\right] e^{-\frac{K_{R}}{\rho_{R} C_{R}} \frac{(2 n-1)^{2} \pi^{2}}{L_{i}^{2}} t},
$$




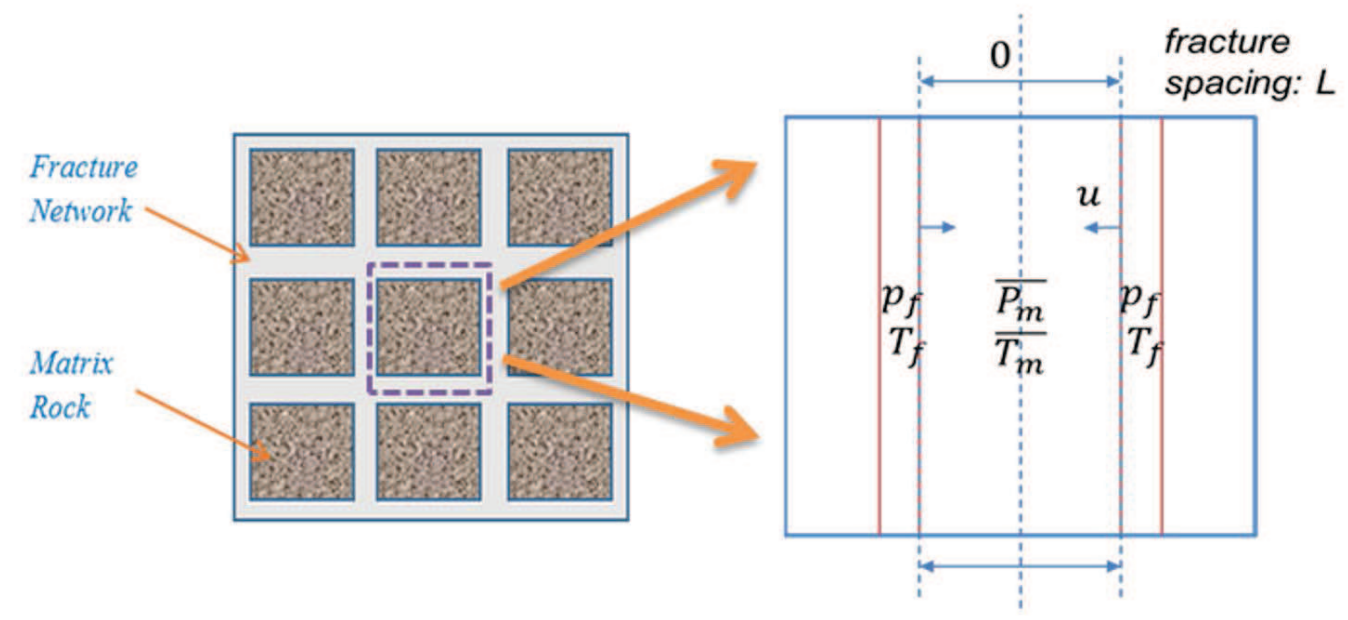

Figure 1: Conceptual model of fracture-matrix network.

where $T_{m i}$ is the initial temperature of the system and $L_{i}$ is the fracture spacing.

We obtain the displacement distribution inside the matrix block using an analytical solution. We rewrite the displacement equation in three dimensions as follows,

$$
\begin{aligned}
& \alpha \frac{\partial P}{\partial x}+3 \beta K \frac{\partial T}{\partial x}+(G+\lambda)\left(\frac{\partial^{2} u_{x}}{\partial x^{2}}+\frac{\partial^{2} u_{y}}{\partial x \partial y}+\frac{\partial^{2} u_{z}}{\partial x \partial z}\right)+G\left(\frac{\partial^{2} u_{x}}{\partial x^{2}}+\frac{\partial^{2} u_{x}}{\partial y^{2}}+\frac{\partial^{2} u_{x}}{\partial z^{2}}\right)+F_{x}=0, \\
& \alpha \frac{\partial P}{\partial y}+3 \beta K \frac{\partial T}{\partial y}+(G+\lambda)\left(\frac{\partial^{2} u_{x}}{\partial x \partial y}+\frac{\partial^{2} u_{y}}{\partial y^{2}}+\frac{\partial^{2} u_{z}}{\partial y \partial z}\right)+G\left(\frac{\partial^{2} u_{y}}{\partial x^{2}}+\frac{\partial^{2} u_{y}}{\partial y^{2}}+\frac{\partial^{2} u_{y}}{\partial z^{2}}\right)+F_{y}=0 \\
& \alpha \frac{\partial P}{\partial z}+3 \beta K \frac{\partial T}{\partial z}+(G+\lambda)\left(\frac{\partial^{2} u_{x}}{\partial x \partial z}+\frac{\partial^{2} u_{y}}{\partial y \partial z}+\frac{\partial^{2} u_{z}}{\partial z^{2}}\right)+G\left(\frac{\partial^{2} u_{z}}{\partial x^{2}}+\frac{\partial^{2} u_{z}}{\partial y^{2}}+\frac{\partial^{2} u_{z}}{\partial z^{2}}\right)+F_{z}=0 .
\end{aligned}
$$

In this work, the thermal changed induced by the leak-off effect is ignored. The 1-D version of the governing displacement equation with respect to matrix block can then be rewritten as

$$
3 \beta K \frac{\partial T}{\partial x}+(2 G+\lambda) \frac{\partial^{2} u_{x}}{\partial x^{2}}=0
$$

At the boundary and the center, the rock displacement satisfies the following boundary conditions,

$$
\begin{aligned}
& \Delta \sigma_{x x}=-k_{f} u_{x}-\Delta P_{f}, \quad x=L_{i} / 2, \\
& u_{x}=0, \quad x=0,
\end{aligned}
$$

where $k_{f}$ is the 'stiffness' of the fracture, which quantifies the resistance of the fracture with respect to thermal-induced displacement. 
With the governing equations and boundary conditions, the displacement at the fracturematrix interface can be obtained as

$$
u_{i}\left(\frac{L_{i}}{2}\right)=\frac{-\left(p_{f}-p_{f, 0}\right)+\beta\left(\overline{T_{m}}-T_{m, 0}\right) \frac{E_{m}}{1-2 v}}{\frac{E_{m}}{1-2 v}+k_{f} L_{i} / 2} \cdot \frac{L_{i}}{2} .
$$

In the above equation, $i$ refers to a certain direction. The fracture aperture change $\Delta b_{i}$ is

$$
\Delta b_{i}=-2 \Delta u_{i}\left(\frac{L_{i}}{2}\right)
$$

Therefore, the fracture aperture change can be expressed as

$$
\Delta b_{i}=\frac{\left(p_{f}-p_{f, 0}\right)-\beta\left(T_{m}-T_{m, 0}\right) \frac{E_{m}}{1-2 v}}{\frac{E_{m}}{1-2 v}+k_{f} L_{i} / 2} \cdot L_{i} .
$$

Fracture permeability can be calculated using the cubic law as

$$
K_{f i}=C_{i} \frac{\left(b_{i 0}+\Delta b_{i}\right)^{2}}{12 L_{i}}
$$

where $C_{i}$ is a parameter to correlate between the mechanical aperture (mechanical opening) and the hydraulic aperture (conductive opening). In this work, $C_{i}$ is set to be 1 , meaning the mechanical opening is fully conductive. The choice of $C_{i}$ will not qualitatively affect our analysis. The correlation between the aperture and permeability can also be applied to cases where the fractures have elastic-plastic resistance. When treated as elastic-plastic material, the fracture has different stiffness within the elastic and plastic zone. When deformation is relatively small, the fracture has elastic behaviors with constant elastic stiffness. The fracture becomes plastic material, when the entire fracture aperture variation exceeds a certain 'breaking' criteria. In this work, the deformation at which fracture breaks is set to be $1 \mathrm{~mm}\left(3.2^{*} 10^{-3} \mathrm{ft}\right)$. Within the elastic zone, the resistance of the fracture is set to be $16 \mathrm{GPa} / \mathrm{m}(250000 \mathrm{psi} / \mathrm{ft}$.). Within the plastic zone, the resistance of the fracture is set to be $1 \mathrm{GPa} / \mathrm{m}$ (16000 psi/ft), as shown in Fig. 2.

With the above fracture properties, for a typical reservoir with a fracture spacing of 20 inches $(0.508 \mathrm{~m})$, the fracture aperture change curve of each grid block is similar to the curve shown in Fig. 3.

As shown in Fig. 3, once the elastic-plastic transition point is reached, the fracture stiffness suddenly drops, resulting in discontinuity of the fracture permeability. The fracture aperture rapidly increases by $200 \%$ to $300 \%$ and exceeds the elastic region. Because of the cubic law, the fracture permeability will increase accordingly by one order of magnitude. The above change of fracture permeability takes place within the time period of less than one day, which is smaller than the usual time step in reservoir simulation.

To simulate the cold water injection problem, we have to deal with a coupled THM problem and so far no satisfactory linear preconditioner has been developed for thermal problems. In this sense we aim to improve the nonlinear solver. 


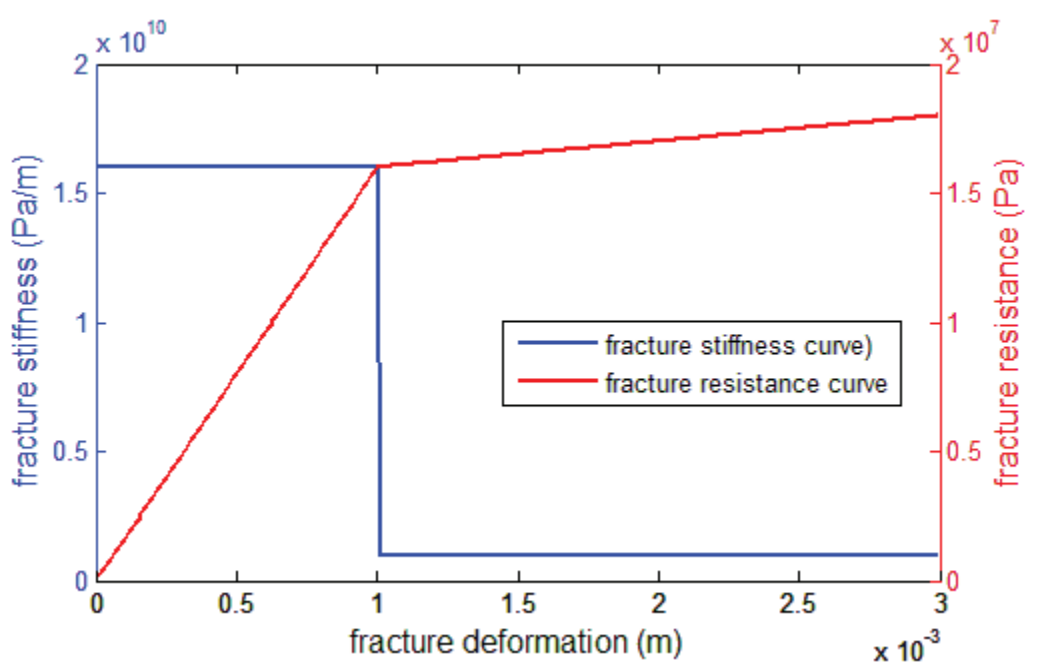

Figure 2: Curve showing the elastic-plastic deformation of the fracture. Blue line: fracture stiffness curve. Red line: fracture resistance curve.

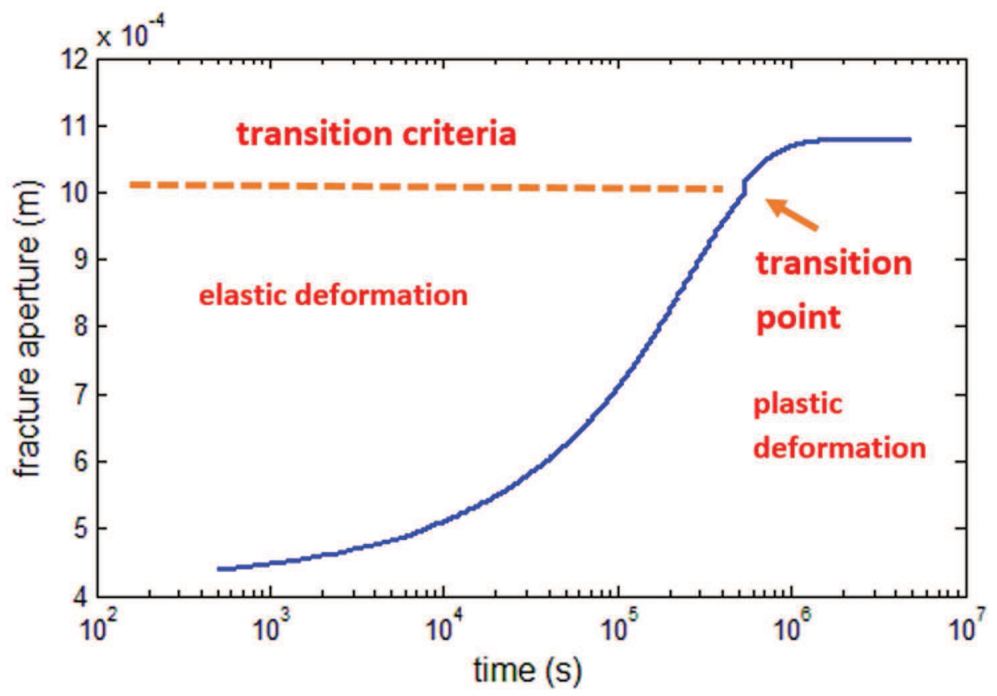

Figure 3: Elastic-plastic fracture aperture change. The arrow indicates the transition point from elastic deformation region to plastic deformation region.

\section{Description of algorithm}

As described above, certain local discontinuity exists in the cold water injection problem. In this section, we describe the nonlinearity elimination Inexact Newton method. Suppose the THM coupled nonlinear system is obtained by rearranging the term in Eq. (2.12), 
as

$$
F(x)=0,
$$

where $F$ is the rearranged governing equation system and $\mathrm{x}$ is the primary variable described in Section 2.1.

\subsection{Restriction of highly nonlinear region}

For each time step, we use the primary variables from the previous time step as the initial guess. Within each time step, local nonlinearity is eliminated before Inexact Newton iteration step to smooth the nonlinear equations. All grid blocks, $S$, are divided into two sets: grid blocks with high nonlinearity $S^{h}$ and grid blocks with low nonlinearity $S^{l}$

$$
S=S^{h} \bigcup S^{l}
$$

Based on the grid blocks, the problem can be divided accordingly into two subdomains as

$$
\begin{aligned}
& V^{h}=\left\{v\left|v=\left(v_{1}, \cdots, v_{n}\right)^{T} \in R^{n}\right| v_{i}=0 \text { if } i \notin S^{h}\right\}, \\
& V^{l}=\left\{v\left|v=\left(v_{1}, \cdots, v_{n}\right)^{T} \in R^{n}\right| v_{i}=0 \text { if } i \notin S^{l}\right\} .
\end{aligned}
$$

Then two restrictors $R_{h}$ and $R_{l}$ are introduced, which restrict the entire system to subdomains with higher nonlinearity and lower nonlinearity respectively. $R_{h}: R^{n} \rightarrow V^{h}$ is a transformation that eliminates the low nonlinearity region and keeps only the high nonlinearity region. $R_{l}: R^{n} \rightarrow V^{l}$, on the other hand, keeps only the low nonlinearity region. In this sense, $R_{h}$ and $R_{l}$ divides the original problem into two subspace problems

$$
\begin{aligned}
& F_{S^{h}}(x)=R_{h} F(x), \\
& F_{S^{l}}(x)=R_{l} F(x) .
\end{aligned}
$$

The formulation of $R_{h}$ and $R_{l}$ is similar to that used in [18].

Then we solve the subspace problem

$$
F_{S^{h}}\left(R_{h} x+C_{h}\right)=0 .
$$

In the above equation, $C_{h}$ is the local correction. Then we combine all subspace corrections and update the primary variables as $x=R_{h} x+C_{h}$.

In this way, the local nonlinearity is eliminated by the above restriction before the start of nonlinear iterations. This local nonlinearity elimination step can be viewed as a preliminary nonlinear preconditioner. As observed by Hwang et al. [18], this preliminary elimination process in average requires much less iterations, compared to the INB step. This step eliminates the local nonlinearity within a small region of the whole domain. The region can be adaptively chosen using physics-based criteria that measures the severity of 
the nonlinearity. In our problem, we use the rock deformation as the criteria, which will be explained in details in the example section. This method can also been used in other situations such as the existence of strong contrast of reservoir properties by changing the criteria for high nonlinearity [25]. Since the number of highly nonlinear grid blocks is typically much smaller compared to the total number of grid blocks, the subspace problem can be simulated by one or two processors and without an elaborate domain partitioning. With the preliminary preconditioner, the nonlinearity can be eliminated more efficiently, as shown below.

\subsection{Inexact Newton with backtracking}

After the preliminary nonlinearity elimination, we solve the resulting nonlinear system using restricted Schwarz Preconditioned Inexact Newton with Backtracking method. We first describe the INB $[17,18]$. Within the $k^{\text {th }}$ iteration, INB solves the system $J\left(x^{k}\right) s^{k}=$ $-R\left(x^{k}\right)$ approximately and update $x^{k}$ as

$$
x^{k}=x^{k}+\lambda^{k} s^{k}
$$

until

$$
\left\|R\left(x^{k}\right)+\lambda^{k} J^{-1}\left(x^{k}\right) R\left(x^{k}\right)\right\| \leq \eta\left\|R\left(x^{k}\right)\right\|,
$$

where $\lambda^{k}$ is the damping (line-search) parameter ranging from 0 to 1 . It shortens the Newton steps to enhance the robustness of the iteration. $\eta$ is the forcing term. If $\eta$ is 0 (or round-off error), INB reduces to exact Newton method. In this work, $\eta$ is set to be constant as $10^{-4}$.

\subsection{Restricted Schwarz preconditioning}

The restricted Schwarz preconditioning step is called between two INB steps. We partition the grid domain into $\mathrm{N}$ non-overlapping subdomains $S_{i}^{0}$,

$$
\bigcup_{i=1}^{N} S_{i}^{0}=S .
$$

The above domain partition can be conducted by existing packages. In this work we use METIS [26]. Then, we define the 1-level overlapping subdomain $S_{i}^{1}$. For $S_{i}^{1}$, every two subdomains overlap with each other with 1-level, which means each subdomain has a 'rim' locating inside its neighboring subdomains.

After defining the subdomains, we can define restrictors $R_{i}^{0}$ and $R_{i}^{1}$ which restricts the nonlinear system on $S$ to a subdomain $S_{i}^{0}$ and $S_{i}^{1}$, respectively. A restrictor drops off the component outside its according subdomain and only keeps those components belonging to the according subdomain. Note here unlike $S^{h}$ and $S^{l}$, these $\mathrm{N}$ subdomains will not adaptively change during the simulation. In this sense, compared to the NE step 
which is of very small scale, the restricted Schwarz preconditioning step is to balance the nonlinearity for massive parallel computing

$$
F_{i}^{1}(x)=R_{i}^{1} F(x)
$$

Each subdomain solves the local problems approximately in parallel using INB method,

$$
F_{i}^{1}\left(x^{k}+v_{i}^{1}\right)=0
$$

where $v_{i}^{1}$ is the correction for subdomain $i$. Neighboring subdomains exchange boundary conditions. In this process the information on the overlapping part of each pair of subdomains is dropped. Such treatment is called the restricted Schwarz method. It has been observed by Cai and Li [27] that the restricted Schwarz method maintains faster convergence rate while has less communication. The formulation of restricted Schwarz method is as follows

$$
x^{k}=\sum_{i=1}^{N} R_{i}^{0}\left(x^{k}+v_{i}^{1}\right)
$$

In this step, the stopping criteria is set as follows. For each type of equation, $j(j=1,2,3,4)$ evaluate the norm of the residual $\left\|R_{1, j}^{1}\left(x^{k}\right)\right\|$ to $\left\|R_{N, j}^{1}\left(x^{k}\right)\right\|$.

Let $\left\|R_{m, j}^{1}\left(x^{k}\right)\right\|=\max \left\{\left\|R_{i, j}^{1}\left(x^{k}\right)\right\| \mid 1 \leq i \leq N\right\}$, therefore $\left\|R_{m, j}^{1}\left(x^{k}\right)\right\|$ is the largest residual of the $j$-th type of equation among all the subdomains. The nonlinearity is balanced only when

$$
\left\|R_{m, j}^{1}\left(x^{k}\right)\right\|<\rho_{j}\left\|R\left(x^{k}\right)\right\| \quad \text { for } j=1,2,3,4 ; \quad m=1,2, \cdots, N
$$

where the parameter $\rho_{j}$ is tolerance, chosen by the user. Based on our observation, between 0.6 to 0.8 yields best performance. In this work, $\rho_{j}$ is set as 0.75 . This nonlinearity balance step can be viewed as a preconditioner that is implemented between two INB steps.

To briefly sum up, our approach implements a nonlinearity elimination preconditioner to preliminarily reduce the local discontinuity and a restricted Schwarz preconditioner called between INB steps to balance the nonlinearity among all processors. The primary purpose of the nonlinearity elimination preconditioner is to improve the convergence performance of the INB method. We aim to achieve a balance between the fast speed of INB method and the good convergence of NR method.

By conducting these operations, we aim to eliminate the effect of local discontinuity from global iterations to improve the robustness of INB. A flowchart of this proposed algorithm showing all operations is as shown in Fig. 4. 


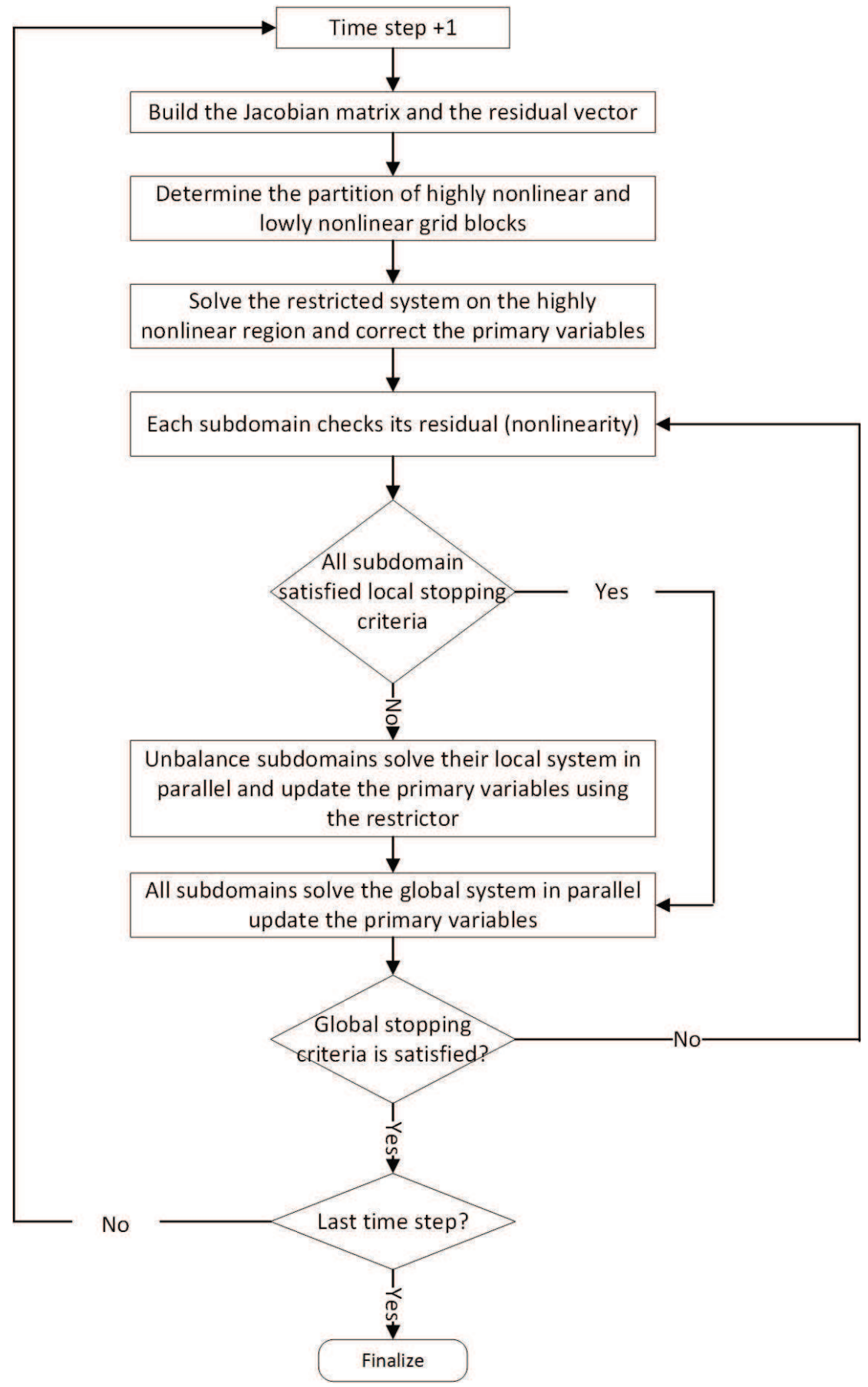

Figure 4: Flowchart of INB-ANE, showing the sequence of all operations within one time step. 


\section{Numerical results}

\subsection{Problem setup}

In this section, we use the proposed preconditioned INB solver to solve a reservoir scale problem. The problem setup of the numerical case is as follows. One cold water injector and one production well locate symmetrically at the middle of the two boundaries of a fractured reservoir. The size of the reservoir is $762.00 \mathrm{~m}^{*} 762.00 \mathrm{~m} * 152.00 \mathrm{~m}$. The reservoir is divided into $80^{*} 80^{*} 15=96000$ structured grid blocks. Cold water is injected for five years at a constant rate of $1.0 \mathrm{~kg} / \mathrm{s}(545 \mathrm{bbl} / \mathrm{d})$ from the injection well. The production well is producing at a constant bottom hole pressure of $3.0 \mathrm{MPa}$. The reservoir is $3 \mathrm{~km}$ in depth. The vertical in-situ stress is estimated from the density of caprock, which is assumed to be sandstone.

Therefore, the vertical in-situ stress $v_{z}$ is $73.50 \mathrm{MPa}$, the minimum horizontal stress $v_{\text {min, hor }}$ and maximum horizontal stress $v_{\text {max }}$,hor are both set to be $51.45 \mathrm{MPa}$, which is $70 \%$ of the vertical in-situ stress. The rock properties are listed in Table 1 . We will investigate the numerical performance of INB-ANE with different combinations of injection temperature, initial fracture permeability and breaking criteria. 8 cases have been run, the input parameters of which are shown in Table 2. Each case is run by 8 processes in parallel. Note here we treat the fracture permeability as an isotropy diagonal vector. In the following simulations, the cap rock is not modelled and the rock properties shown in Table 1 are for fractured rock only.

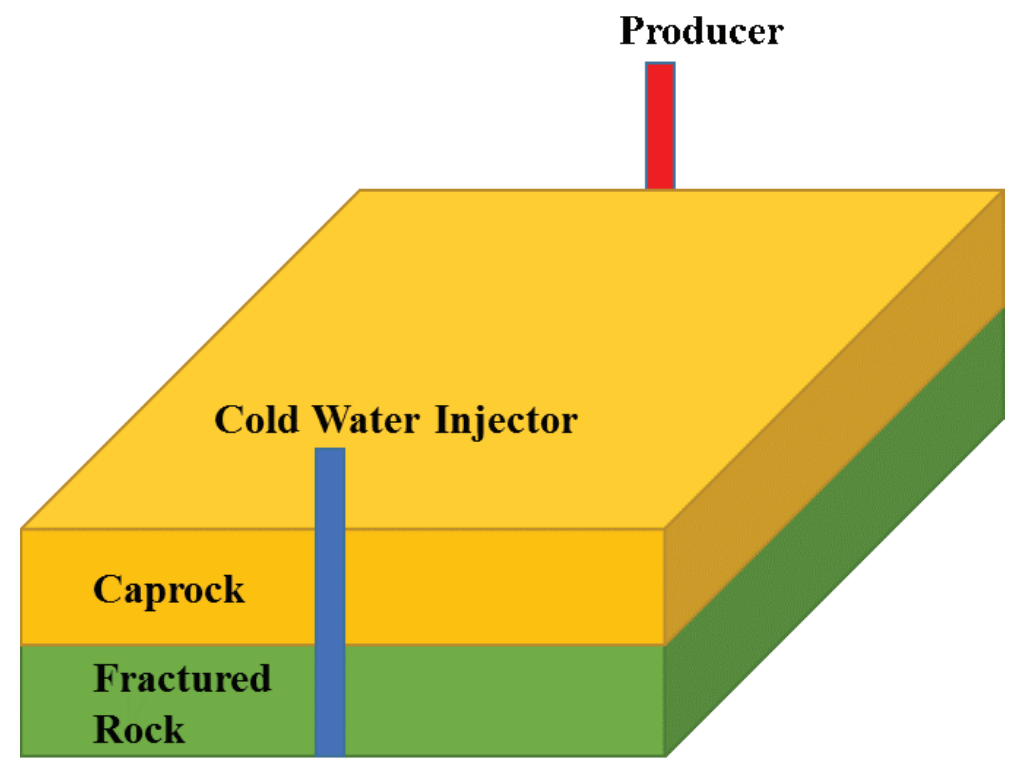

Figure 5: Conceptual model of the cold water injection problem. 
Table 1: List of input properties of the rock.

\begin{tabular}{||l|l|l||}
\hline Properties & Values & Units \\
\hline Initial permeability of the matrix & $\begin{array}{l}K_{m x}=K_{m y}=1.0^{*} 10^{-15} \\
K_{m z}=1.0^{*} 10^{-15}\end{array}$ & $\mathrm{~m}^{2}$ \\
\hline Initial porosity of the matrix & 0.15 & dimensionless \\
\hline Initial porosity of the fracture & 0.001 & dimensionless \\
\hline Young's modulus & 16.0 & $\mathrm{GPa}$ \\
\hline Fracture spacing & 0.5 & $\mathrm{~m}$ \\
\hline Poisson's ratio & 0.25 & dimensionless \\
\hline Biot's coefficient & 1.0 & dimensionless \\
\hline Initial pore pressure & 25.0 & $\mathrm{MPa}$ \\
\hline Initial temperature & 368 & $\mathrm{~K}$ \\
\hline Linear thermal expansion coefficient & 11.6 & $10^{-6} \mathrm{~m} /(\mathrm{m} \cdot \mathrm{K})$ \\
\hline Thermal conductivity of dry rock & 1.0 & $\mathrm{~W} /(\mathrm{m} \cdot \mathrm{K})$ \\
\hline Heat capacity of rock & 1000 & $\mathrm{~J} /(\mathrm{kg} \cdot \mathrm{K})$ \\
\hline Density of rock & 2.5 & $103 \mathrm{~kg} / \mathrm{m}^{3}$ \\
\hline$v_{z}$ & 73.50 & $\mathrm{MPa}$ \\
\hline$v_{m i n}$ hor & 51.45 & $\mathrm{MPa}$ \\
\hline Elastic resistance & 12.0 & $\mathrm{GPa}$ \\
\hline Plastic resistance & 0.7 & $\mathrm{GPa}$ \\
\hline
\end{tabular}

Table 2: Input parameters for Case 1 to 8.

\begin{tabular}{||l|l|l|l||}
\hline Case index & $\begin{array}{l}\text { Injection } \\
\text { Temperature }(\mathrm{K})\end{array}$ & $\begin{array}{l}\text { Initial fracture } \\
\text { permeability }\left(\mathrm{m}^{2}\right)\end{array}$ & $\begin{array}{l}\text { Breaking criteria } \\
\text { (transition aperture) }\left({ }^{*} 10^{-6}\right)\end{array}$ \\
\hline 1 & 288.00 & $1.00^{*} 10^{-11}$ & 200.00 \\
\hline 2 & 288.00 & $1.00^{*} 10^{-11}$ & 250.00 \\
\hline 3 & 288.00 & $5.00^{*} 10^{-12}$ & 200.00 \\
\hline 4 & 288.00 & $5.00^{*} 10^{-12}$ & 250.00 \\
\hline 5 & 298.00 & $1.00^{*} 10^{-11}$ & 200.00 \\
\hline 6 & 298.00 & $1.00^{*} 10^{-11}$ & 250.00 \\
\hline 7 & 298.00 & $5.00^{*} 10^{-12}$ & 200.00 \\
\hline 8 & 298.00 & $5.00^{*} 10^{-12}$ & 250.00 \\
\hline
\end{tabular}

\subsubsection{Criteria for determining high nonlinearity zone}

The nonlinearity elimination criteria determines the high nonlinearity zone. In this problem, the nonlinearity is mainly the local discontinuity introduced by the failure of the 
fractured material. We set the nonlinearity elimination criteria based on the relationship between the deformation (aperture change) $\Delta b$ and the critical (breaking) deformation $\Delta b_{c}$ of the fractured material as follows. For a given grid block $v$, suppose

$$
\Delta b(v)=\theta \Delta b_{c}
$$

where $\theta$ is a dynamically changing value.

The judgment that whether $v$ belongs to the high nonlinearity region is made as:

$$
\begin{cases}v \in S^{h}, & \text { if } \theta(v) \in(0.4,1.2), \\ v \in S^{l}, & \text { otherwise. }\end{cases}
$$

We adopt Case 1 as an example. For this case, the injection temperature is $288 \mathrm{~K}$ and the breaking deformation $\Delta b_{c}$ is $200 \mu \mathrm{m}$. The initial fracture aperture can be calculated as $350 \mu \mathrm{m}$. Then according to Eq. (3.12), a grid block belongs to the high nonlinearity group if its aperture change is between $430 \mu \mathrm{m}$ to $590 \mu \mathrm{m}$. For the grid block locating at the injection point in Case 1, its fracture aperture change curve is shown in Fig. 6. According to Fig. 6 , the grid block belongs to the high nonlinearity region between $1.0^{6} \mathrm{~s}$ (11.5 days) to $7.3 * 10^{6} \mathrm{~s}$ ( 84.5 days). We plot the permeability field at 180 days and 7 years after the start of the injection in Fig. 7. Moreover, we plot the according distribution of the high/low nonlinearity regions in Fig. 8. By comparing Fig. 7 and Fig. 8, we can see that as the permeability enhancement effect propagates, the highly nonlinearity region is around the heat front region. Besides, in either time point, the highly nonlinearity region only takes a small portion of the entire domain.

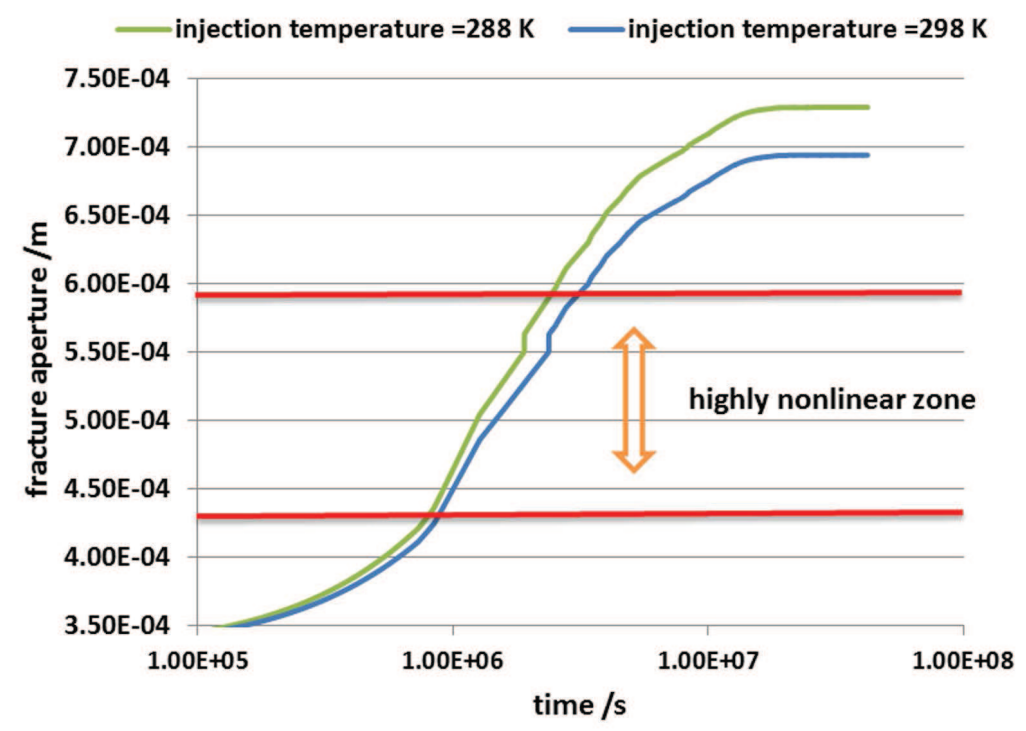

Figure 6: Fracture aperture curve at the cold water injector of Case 1 and Case 5. Red lines indicate the highly nonlinear zone determined by the judging criteria. 

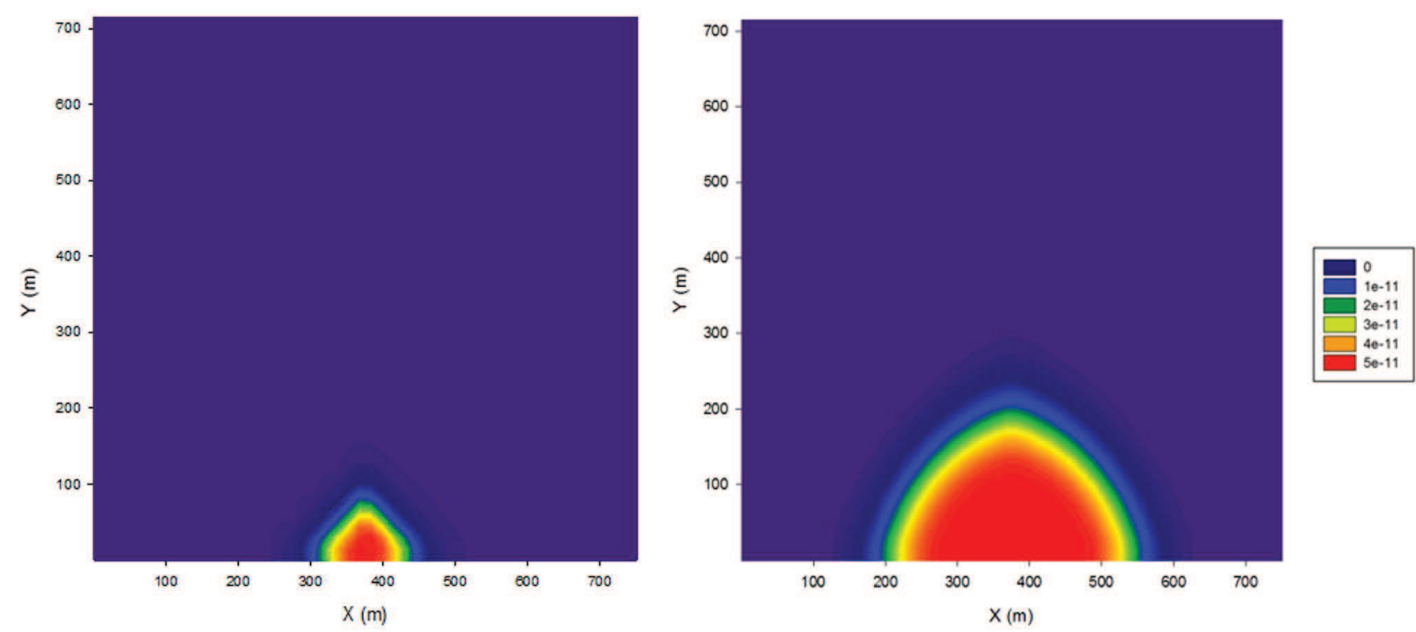

Figure 7: Permeability field of Case 1 after cold water injection. The unit of the fracture permeability is $m^{2}$. Red/orange color indicates the permeability enhanced zone near the injector. The figure on the left is the ydirection permeability field at 180 days of injection, while the figure on the right is the y-direction permeability field after seven years of injection. In this case, the initial fracture permeability $10^{-11} \mathrm{~m}^{2}$ (10 Darcy).
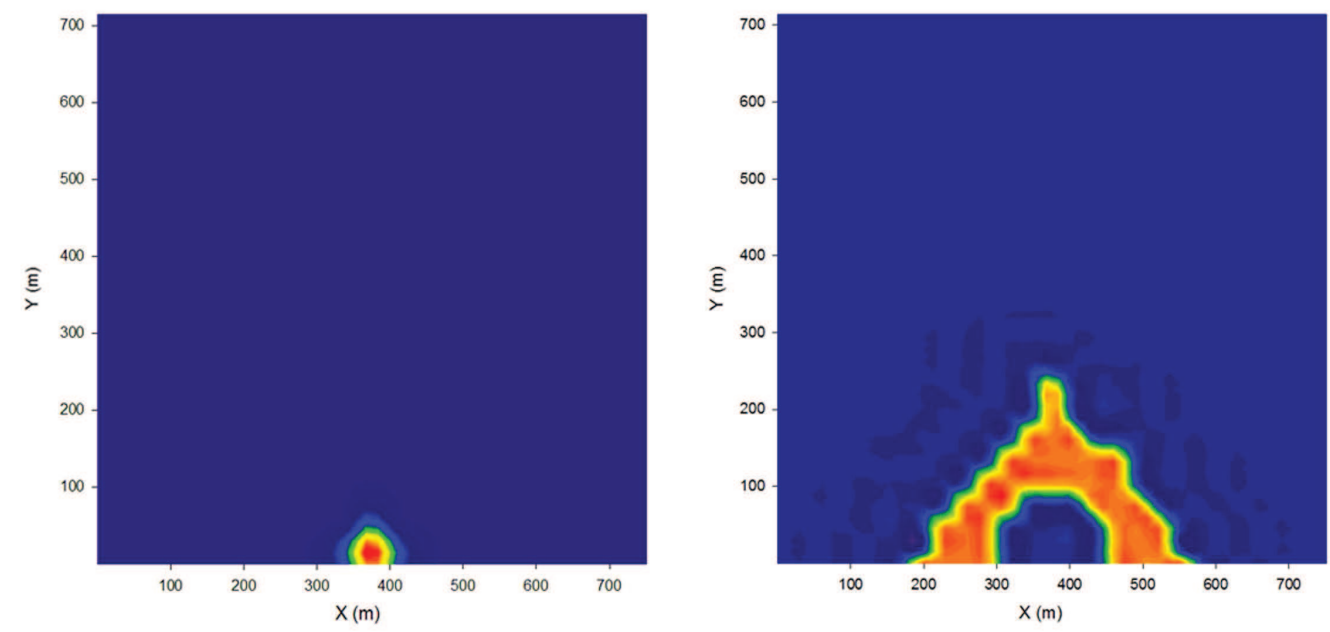

Figure 8: Distribution of high/low nonlinearity regions of Case 1. Red/orange color indicates the high nonlinearity region that is determined by the physics-based judging criteria. Blue color indicates the low nonlinearity region. The figure on the left is the distribution at 180 days of injection, while the figure on the right is that at seven years of injection.

\subsection{Numerical performance}

In this section, we present the numerical performance of the proposed method. We first compare the speed of the convergence of INB, IBN-ANE and NR method. In this work, we set that maximum number of allowed iterations within one time step to be 10 . If it 


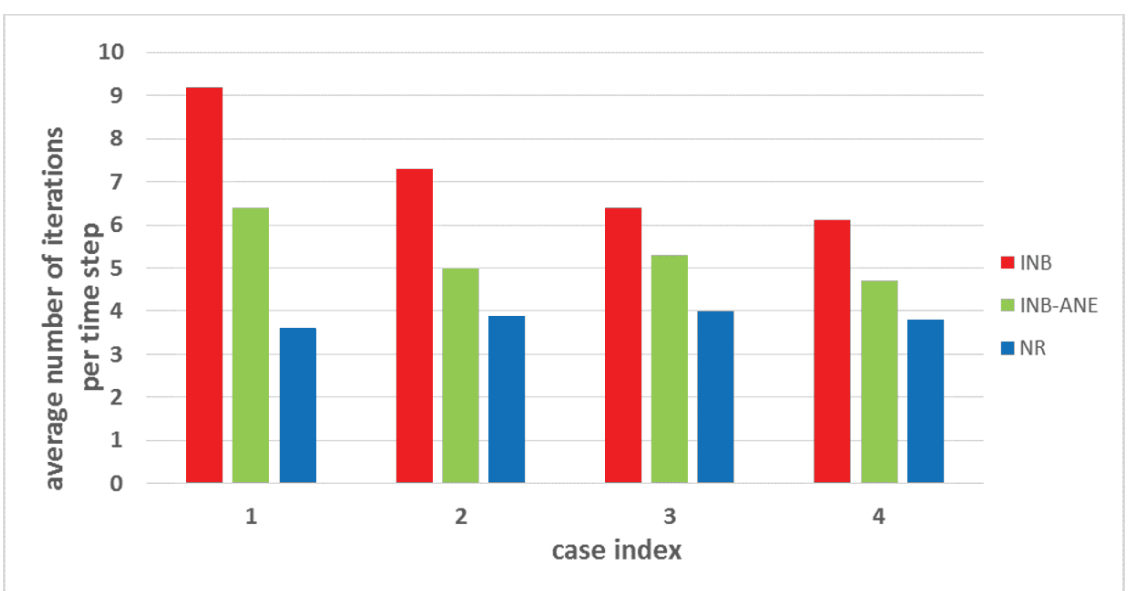

Figure 9: Comparison of the average number of iterations per time step of Case 1 to Case 4. Red columns: Inexact Newton Method without preconditioning. Green columns: the proposed INB-ANE method. Blue columns: Newton-Raphson method.

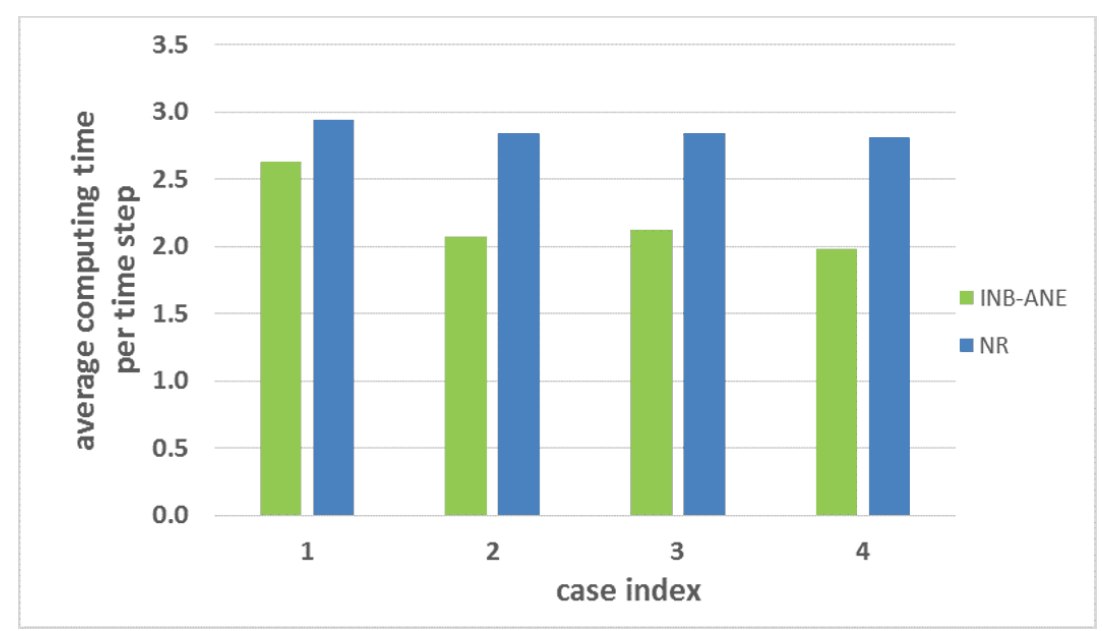

Figure 10: Comparison of the average computing time per time step of Case 1 to Case 4. Green columns: the proposed INB-ANE method. Blue columns: Newton-Raphson method.

requires more than 10 iterations to converge, the iteration is terminated and the time step is cut. For NR method, it iterates until the $L 2$ norm of the relative residual is smaller than $10^{-6}$. The average number of iterations per time step and the average on-wall computing time per time step are shown in Figs. 9-12. As the results demonstrate, the average number of iterations per time step of INB-ANE is in the range to 4 to 6 , comparable to that of NR method and much less than of INB without preconditioning. It turns out that by implementing such a preconditioner, INB becomes more efficient and more robust. As INB type of method requires less time spent in the linear solver within each iteration, the pro- 


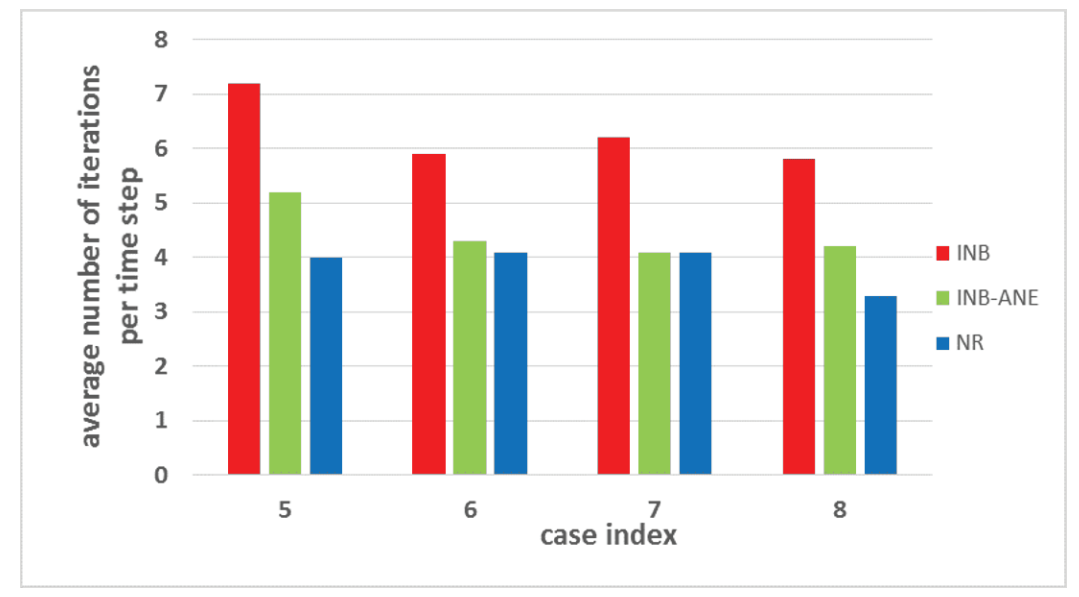

Figure 11: Average number of iterations per time step of Case 5 to Case 8. Red columns: Inexact Newton Method without preconditioning. Green columns: the proposed INB-ANE method. Blue columns: NewtonRaphson method.

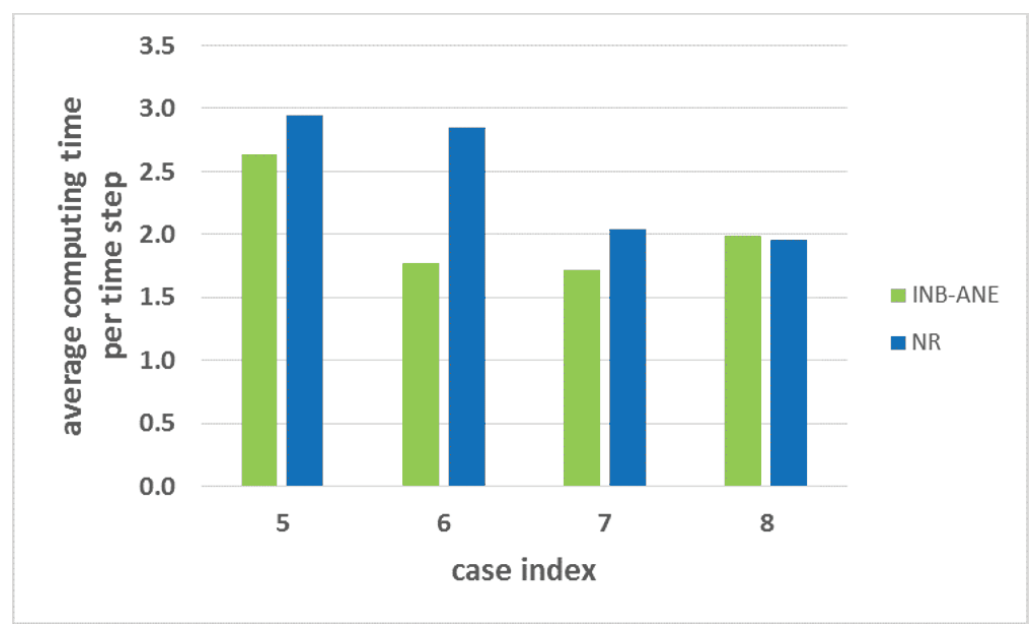

Figure 12: Comparison of the average computing time per time step of Case 5 to Case 8. Green columns::the proposed INB-ANE method. Blue columns:Newton-Raphson method.

posed INB-ANE method can accelerate the simulation by $10 \%$ to $30 \%$, as demonstrated by Fig. 10 and Fig. 12.

\subsection{Parallel performance}

Parallel performance is a critical criteria to evaluate of nonlinear solvers. In this subsection, we investigate the parallel speedup factor of INB-ANE and compare it with that of NR method. We re-run Case 1 with the same input parameters but on a refined grid with $256^{*} 256 * 20=1,310,720$ uniform grid blocks. Since the parallel performance is close 
Table 3: Comparison of the parallel speedup factor of INB-ANE and NR.

\begin{tabular}{||l|l|l|l|l|l||}
\hline $\begin{array}{l}\text { Number of } \\
\text { processors }\end{array}$ & $\begin{array}{l}\text { Time spent by } \\
\text { INB-ANE (s) }\end{array}$ & $\begin{array}{l}\text { Speedup factor } \\
\text { for INB-ANE }\end{array}$ & $\begin{array}{l}\text { Time spent } \\
\text { by NR(s) }\end{array}$ & $\begin{array}{l}\text { Speedup factor } \\
\text { of NF }\end{array}$ & $\begin{array}{l}\text { Ideal(linear) } \\
\text { speedup }\end{array}$ \\
\hline 2 & 62490.62 & 2 & 77182.2 & 2 & 2 \\
\hline 4 & 32554.01 & 3.82 & 39990.78 & 3.86 & 4 \\
\hline 8 & 17058.48 & 7.29 & 20581.92 & 7.50 & 8 \\
\hline 16 & 9273.40 & 13.41 & 11026.03 & 14.01 & 16 \\
\hline 32 & 4892.06 & 25.42 & 5629.62 & 27.5 & 32 \\
\hline 64 & 2952.42 & 42.12 & 3207.90 & 48.12 & 64 \\
\hline 128 & 1778.552 & 69.92 & 1946.09 & 79.32 & 128 \\
\hline 256 & 1137.544 & 109.33 & 1244.87 & 123.32 & 256 \\
\hline
\end{tabular}

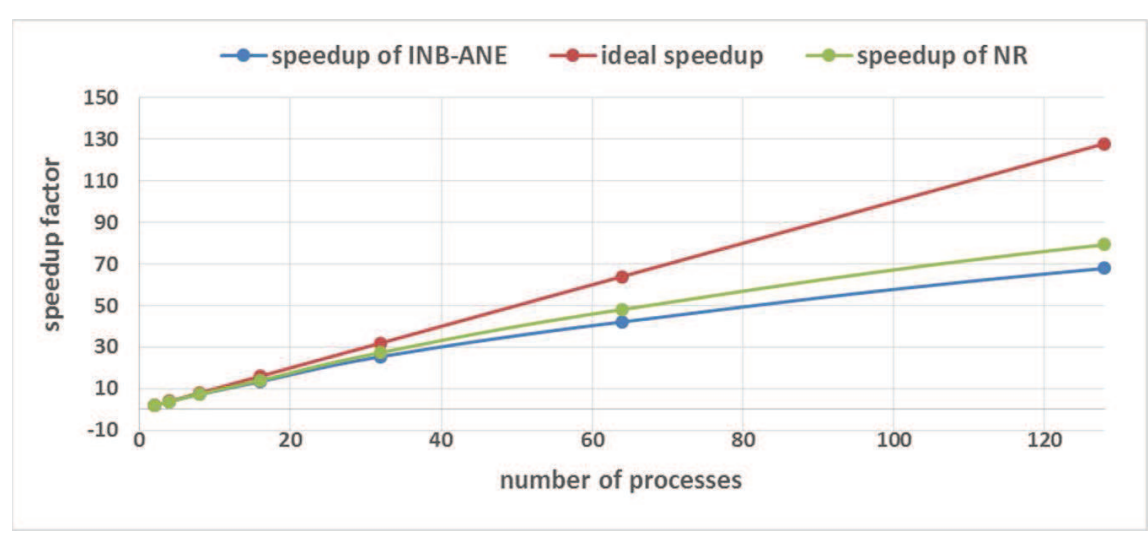

Figure 13: Comparison of the parallel speedup between INB-ANE, NR, and ideal performance.

to linear speedup when the number of processors is low enough, we use the parallel performance of the 2-processor cases as the benchmark.

As demonstrated by Table 3 and Fig. 13, INB-ANE is nonlinearly scalable. Compared to Newton-Raphson method, the speedup factor of INB-ANE deteriorates faster when the number of processors increases, which is mainly due to the fact that the preliminary nonlinearity elimination step is not fully scalable. However, it should be noticed that when the number of processes increases to 256, INB-ANE still increases the speed by around $10 \%$.

\section{Conclusions}

We have developed a nonlinearly preconditioned Inexact Newton Method, INB-ANE. The proposed method dynamically eliminates and balances the local nonlinearity and 
discontinuity. The method adopts physics-based criteria to determine the high nonlinear zone within the computing domain. With algebraic restrictors, the method can be easily implemented into the codes of existing massively parallel simulators.

We have used INB-ANE to simulate the coupled thermal-hydraulic-mechanical behaviors of fractured reservoirs with elastic-plastic deformations of the fractures induced by cold water injection. It has been found that the proposed method can improve the robustness of the Inexact Newton method, avoiding iteration failures or time step cuts. Compared to commonly used Newton-Raphson method, the proposed method can save $10 \%$ to $30 \%$ of the computing time, depending on the input parameters and the number of processors used.

The proposed INB-ANE method is a competitive nonlinear solver in simulating the tightly coupled multi-physics problems with highly local nonlinearity in the practice of reservoir simulation. Its application can be extended to reservoir optimization [28] as well as uncertainty qualification [29]. It can also be used to the integration of the simulation of hydraulic fracturing and reservoir simulation [30], in which the fracture front is the highly nonlinear region.

\section{Acknowledgments}

The author would like to thank the support of CNPC-USA and Chuanqing Downhole Service Company.

\section{References}

[1] Keyes D E, McInnes L C, Woodward C, et al. Multiphysics Simulations: Challenges And Opportunities. The International Journal of High Performance Computing Applications, 27 (2013), 483.

[2] Wang S, Xiong Y, Winterfeld P, et al. Parallel Simulation Of Thermal-Hydrological-Mechanic (THM) Processes In Geothermal Reservoirs, in: Proc. 39th Work. Geotherm. Reserv. Eng. Stanford Univ. Stanford, Calif., 2014.

[3] Wang S, Huang Z, Wu Y-S, et al. A Semi-Analytical Correlation Of Thermal-HydraulicMechanical Behavior Of Fractures And Its Application To Modeling Reservoir Scale Cold Water Injection Problems In Enhanced Geothermal Reservoirs. Geothermics, 64 (2016), 8195.

[4] Dembo R S, Eisenstat S C, Steihaug T. Inexact Newton Methods. SIAM Journal on Numerical Analysis, 19 (1982), 400408.

[5] Eisenstat S C, Walker H F. Choosing The Forcing Terms In An Inexact Newton Method. SIAM Journal on Scientific Computing, 17 (1996), 1632.

[6] Wang S, Winterfeld P H, Wu Y-S. An Efficient Adaptive Nonlinearity Elimination Preconditioned Inexact Newton Method For Parallel Simulation Of Thermal-Hydraulic-Mechanical Processes In Fractured Reservoirs, in: SPE Reserv. Simul. Symp., Society of Petroleum Engineers, 2015.

[7] Kelley C. Iterative Methods For Linear And Nonlinear Equations. Society for Industrial and Applied Mathematics, 1995. 
[8] Eisenstat S C, Walker H F. Globally Convergent Inexact Newton Methods. SIAM Journal on Optimization, 4 (1994), 393422.

[9] Birgin E G, Kreji N, Martnez J M. Globally Convergent Inexact Quasi-Newton Methods For Solving Nonlinear Systems. Numerical Algorithms, 32 (2003), 249260.

[10] Knoll D A, Keyes D E. Jacobian-Free NewtonKrylov Methods: A Survey Of Approaches And Applications. Journal of Computational Physics, 193 (2004), 357397.

[11] Cai X-C, Keyes D E. Nonlinearly Preconditioned Inexact Newton Algorithms. SIAM Journal on Scientific Computing, 24 (2002), 183200.

[12] Griebel M, Oswald P. On The Abstract Theory Of Additive And Multiplicative Schwarz Algorithms. Numerische Mathematik, 70 (1995), 163180.

[13] Cai X-C, Sarkis M. A Restricted Additive Schwarz Preconditioner For General Sparse Linear Systems. Siam Journal on Scientific Computing, 21 (1999), 792797.

[14] Skogestad J O, Keilegavlen E, Nordbotten J M. Domain Decomposition Strategies For Nonlinear Flow Problems In Porous Media. Journal of Computational Physics, 234 (2013), 439451.

[15] Liu L, Keyes D E, Sun S. Fully Implicit Two-Phase Reservoir Simulation With The Additive Schwarz Preconditioned Inexact Newton Method, in: SPE Reserv. Charact. Simul. Conf. Exhib., Society of Petroleum Engineers, 2013.

[16] Yang H, Yang C, Sun S. Active-Set Reduced-Space Methods With Nonlinear Elimination For Two-Phase Flow Problems In Porous Media. Siam J. Sci. Comput., 38 (2016), 593618.

[17] Hwang F-N, Lin H-L, Cai X-C. Two-Level Nonlinear Elimination Based Preconditioners For Inexact Newton Methods With Application In Shocker Duct Flow Calculation. Electronic Transactions on Numerical Analysis, 37 (2010), 239251.

[18] Hwang F-N, Su Y-C, Cai X-C. A Parallel Adaptive Nonlinear Elimination Preconditioned Inexact Newton Method For Transonic Full Potential Equation. Computers \& Fluids, 110 (2015), 96107.

[19] Wu Y. MSFLOW: Multiphase Subsurface Flow Model Of Oil, Gas And Water In Porous And Fractured Media With Water Shut-Off Capability, Documentation and User's Guide. Walnut Creek, California: 1998.

[20] Wang S. Numerical Study Of Thermal-Hydraulic-Mechanical Behavior Of Fractured Geothermal Reservoirs. Colorado School of Mines, 2015.

[21] Ghassemi A, Kumar G S. Changes In Fracture Aperture And Fluid Pressure Due To Thermal Stress And Silica Dissolution/Precipitation Induced By Heat Extraction From Subsurface Rocks. Geothermics, 36 (2007), 115140.

[22] Rutqvist J, Stephansson O. The Role Of Hydromechanical Coupling In Fractured Rock Engineering. Hydrogeology Journal, 11 (2003), 740.

[23] Kim J, Sonnenthal E L, Rutqvist J. Formulation And Sequential Numerical Algorithms Of Coupled Fluid/Heat Flow And Geomechanics For Multiple Porosity Materials. International Journal for Numerical Methods in Engineering, 92 (2012), 425456.

[24] Carslaw H S, Jaeger J C. Conduction Of Heat In Solids. 2nd ed. Oxford: 1959.

[25] Deng S, Jiang R, Karimi-Fard M, et al. Fluid-Flow Effects Of Compartmentalized Distribution Of Compaction Bands In An Aeolian Sandstone In Three Dimensions. Petroleum Geoscience, 23 (2017), 223230.

[26] Karypis G, Kumar V. Metis-Parmetis: Unstructured Graph Partitioning And Sparse Matrix Ordering System, Version 4.0.3. 2009.

[27] Cai X-C, Li X. Inexact Newton Methods With Restricted Additive Schwarz Based Nonlinear Elimination For Problems With High Local Nonlinearity. SIAM Journal on Scientific Com- 
puting, 33 (2011), 746762.

[28] Ampomah W, Balch R S, Grigg R B, et al. Co-Optimization Of CO2-EOR And Storage Processes In Mature Oil Reservoirs. 2016.

[29] Jiang R, Stern D, Halsey T C, et al. Scenario Discovery Workflow For Robust Petroleum Reservoir Development Under Uncertainty. International Journal for Uncertainty Quantification, 6 (2016), 533559.

[30] Tang H, Winterfeld P H, Wu Y-S, et al. Integrated Simulation Of Multi-Stage Hydraulic Fracturing In Unconventional Reservoirs. Journal of Natural Gas Science and Engineering, 36 (2016), 875892. 